\title{
Inability of immunohistochemistry to predict clinical outcomes of endometrial cancer patients
}

\author{
D. R. GOSSETT*, P. ALO†, R. E. BRISTOW \\ \& F. J. MONTZ \\ *The University of Michigan Medical Center, Department of Gynecologic Oncology, Ann Arbor, MI; +University of Rome, \\ Department of Anatomic Pathology, Rome, Italy; ¥The Johns Hopkins Hospital and Medical Institutions, Kelly Gynecologic \\ Oncology Service, Baltimore, MD; §University of Rome, Department of Gynecology, Rome, Italy; 9 Oncotech, Inc., Tustin, CA
}

Abstract. Gossett DR, Alo P, Bristow RE, Galati M, Kyshtoobayeva A,
Fruehauf J, Montz FJ. Inability of immunohistochemistry to predict clinical
outcomes of endometrial cancer patients. Int J Gynecol Cancer 2004;14:145-151.

Introduction: Despite optimal surgery, some patients with early endometrial carcinoma develop recurrence and die of disease. A number of immunohistochemical (IHC)-identified cell products (markers) have been proposed as predictors of recurrence. This study characterizes a large series of endometrial carcinomas with previously described markers as well as markers that have not been investigated in endometrial carcinoma. Patients and methods: Women who had undergone surgery for endometrial carcinoma were identified and specimens accessed. Tissue blocks were evaluated for ten IHC markers. Results were correlated with last known clinical status.

Results: Mean follow-up was 43 months; complete data were available on 117 patients. Two women died of other causes; of the remaining 115, eight died of disease and six were alive with recurrence at last follow-up (12\%). Vascular endothelial growth factor staining independently predicted recurrence and death. However, in multivariate analyses, only FIGO stage predicted outcome.

Discussion: Our goal was to identify markers to predict which women with endometrial carcinoma were likely to have disease recurrence. We evaluated cell-cycle regulatory proteins, growth factors, hormone receptors, and angiogenic factors, but did not identify any marker that independently predicted outcome in multivariate analysis. This may reflect the few negative outcomes in our population.

KEYWORDS: endometrial cancer, immunohistochemistry, VEGF.

Endometrial carcinoma is the most common gynecologic malignancy affecting American women, accounting for an estimated 39,300 new cases and

Address correspondence and reprint requests to: Dana R. Gossett, MD, Department of Obstetrics and Gynecology, The University of Michigan Medical Center, 1500 E. Medical Center Drive, L4510 WH, Ann Arbor, MI 48109-0276. Email: danagoss@med.umich.edu
6600 deaths in the year $2002^{(1)}$. Despite 'definitive' surgical therapy, many women with early stage endometrial carcinoma go on to have recurrence and die of disease. Known risk factors for progression include specific histologic cell types, high histologic grade, deep myometrial invasion, and presence of lymphvascular involvement. However, some women even with apparent grade 1, stage I endometrioid type tumors will experience recurrence. To date, there are no known clinical or pathologic characteristics that 
identify which patients are at highest risk for recurrence and death.

The past decade has seen rapid advances in research on and clinical application of immunohistochemical (IHC) markers in a variety of malignant and non-malignant diseases. Steroid hormones and their receptors, oncogene products, and tumor suppressor gene products have all been investigated as possible predictors of tumor behavior as well as targets for therapy. Characterization of estrogen and progesterone receptor status in breast carcinomas is now standard care and serves to direct adjuvant therapies. More recently, monoclonal antibodies against the HER2/neu receptor in women with HER2/neu overexpression have been included in standard adjuvant therapy of breast cancer ${ }^{(2,3)}$.

In the present study, we examine the prevalence of ten IHC markers in endometrial carcinomas, and correlate them with patient characteristics and outcome data (Table1). The markers can be broadly divided into three general groups: (1) cell-cycle regulatory proteins (tumor suppressor genes), (2) factors associated with cell-cell adhesion and angiogenesis, and (3) steroid hormone receptors. Each category of cellular product is thought to be dysregulated in solid tumors: loss of expression of tumor suppressor genes is a key permissive step in the uncontrolled cell division of malignancy; neovascularization is cri- tical to both local growth and metastasis of solid tumors; and steroid hormones play key roles in regulating proliferation and differentiation of the endometrium. Each of the markers studied could therefore possibly predict more malignant behavior if its expression is abnormal, and could also serve as a future target for molecularly tailored therapy.

Previous studies have suggested that ER/PR negativity $^{(4-6)}$, overexpression of HER2/neu ${ }^{(7)}$, vascular

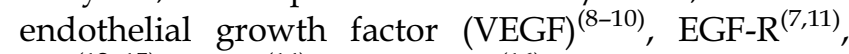
p53 ${ }^{(12-15)}, \mathrm{Ki}-67^{(14)}$, and TSP-1 ${ }^{(16)}$ may predict more malignant behavior of endometrial carcinomas, as reflected by advanced stage or grade, depth of invasion, or lymphvascular space involvement. However, clinical outcomes of patients have not been universally addressed in these investigations.

Additional studies have correlated overexpression of HER2/neu with poorer outcomes in breast and ovarian cancer ${ }^{(17-20)}$. Investigations in breast carcinoma have also associated clinical outcome with markers for angiogenesis such as $\mathrm{CD} 31^{(21)}, \mathrm{CD} 105^{(22,23)}$, and TSP-1 ${ }^{(24)}$. To our knowledge, CD31 and CD105 have not previously been studied in endometrial carcinomas.

We hypothesize that the identification of new markers capable of predicting disease recurrence will allow gynecologic oncologists to tailor both adjuvant therapy and future surveillance for women with early stage endometrial cancers.

Table 1. Immunohistochemical markers

\begin{tabular}{|c|c|c|c|}
\hline Marker & Class & Function & Role in tumorigenesis \\
\hline p53 & Tumor suppressor gene & $\begin{array}{l}\text { Arrests cell cycle for DNA } \\
\text { repair, initiates apoptosis }\end{array}$ & $\begin{array}{l}\text { Loss of wild-type allows uninhibited } \\
\text { growth }\end{array}$ \\
\hline Ki67 & Nuclear protein & $\begin{array}{l}\text { Expressed in normal/neoplastic } \\
\text { tissues throughout cell cycle } \\
\text { except G0 }\end{array}$ & Reflects proliferative/mitotic activity \\
\hline HER2/neu & Oncogene product & $\begin{array}{l}\text { Membrane glycoprotein, partial } \\
\text { homology to EGF-R }\end{array}$ & $\begin{array}{l}\text { Involved in cell-cell adhesion } \\
\text { Facilitates growth, invasion, and metastasis }\end{array}$ \\
\hline EGF-R & Oncogene product & $\begin{array}{l}\text { Promotes cell division, binds } \\
\text { EGF and TGF- } \alpha\end{array}$ & $\begin{array}{l}\text { Overexpression promotes excessive growth } \\
\text { Up-regulated by estrogen }\end{array}$ \\
\hline VEGF & Oncogene product & $\begin{array}{l}\text { Mitogen for vascular } \\
\text { endothelial cells }\end{array}$ & $\begin{array}{l}\text { Promotes angiogenesis in normal } \\
\text { and neoplastic endometrium }\end{array}$ \\
\hline CD31 & $\begin{array}{l}\text { Cell surface protein } \\
\text { specific to vascular } \\
\text { endothelium }\end{array}$ & $\begin{array}{l}\text { Regulates complex signaling } \\
\text { pathway; modulates cell adhesion }\end{array}$ & $\begin{array}{l}\text { Role in promoting angiogenesis } \\
\text { reported in breast carcinomas }\end{array}$ \\
\hline CD105 & $\begin{array}{l}\text { Cell surface protein } \\
\text { specific to vascular } \\
\text { endothelium }\end{array}$ & $\begin{array}{l}\text { TGF- } \beta \text { receptor; modulates } \\
\text { TGF- } \beta \text { signaling }\end{array}$ & $\begin{array}{l}\text { Binds TGF- } \beta \text {, required for angiogenesis; } \\
\text { role in angiogenesis reported in } \\
\text { breast carcinomas }\end{array}$ \\
\hline TSP-1 & $\begin{array}{l}\text { Matrix-bound adhesive } \\
\text { glycoprotein }\end{array}$ & $\begin{array}{l}\text { Regulates cell adhesion, } \\
\text { activates TGF- } \beta\end{array}$ & $\begin{array}{l}\text { May promote metastatic spread and } \\
\text { angiogenesis }\end{array}$ \\
\hline ER & $\begin{array}{l}\text { Steroid hormone } \\
\text { receptor }\end{array}$ & Wide range of regulatory functions & $\begin{array}{l}\text { Uncertain; complex regulatory effect } \\
\text { on many other pathways }\end{array}$ \\
\hline PR & $\begin{array}{l}\text { Steroid hormone } \\
\text { receptor }\end{array}$ & Wide range of regulatory functions & $\begin{array}{l}\text { Uncertain; complex regulatory effect on many } \\
\text { other pathways }\end{array}$ \\
\hline
\end{tabular}

VEGF, vascular endothelial growth factor. 


\section{Patients and methods}

Approval of the investigation was obtained from the Institutional Review Boards of all participating institutions. One hundred and forty-seven women with a pathologic diagnosis of endometrial cancer were retrospectively identified. All patients underwent surgical staging at the Hospital of the University of Rome between 1996 and 2000. All slides were independently re-reviewed by a single pathologist (PA) to verify the diagnosis, histologic subtype, and grade. No significant discrepancies were noted between the original pathologic diagnoses and the diagnoses at review. Clinical data collected included age at diagnosis, existence of common co-morbidities, use of exogenous sex steroids, surgical stage of disease, results of pathologic evaluation, adjuvant therapy received, recurrence and interval to such, and last known status/ interval to death.

\section{Immunohistochemistry}

IHC staining was performed by Oncotech Inc. (Tustin, CA) on fixed, paraffin-embedded tissue blocks. Sections $3-4 \mu \mathrm{m}$ thick were deparaffinized in Histoclear, and rehydrated in descending grades (100-70\%) of ethanol. Antigen retrieval included proteinase $\mathrm{K}$ or protease digestion for CD31, CD105, VEGF, and EGF-R, and microwaving for $20 \mathrm{~min}$ at $5 \mathrm{~min}$ intervals for the p53, PR, HER2/neu, and Ki67 procedures. Slides were kept in a pressure cooker for $20 \mathrm{~min}$ to retrieve ER antigen. No antigen retrieval was necessary for TSP-1 detection. Automated IHC procedures were performed using I6000 (BioGenex, San Ramon, CA) and NexES (Ventana, Phoenix, AZ) immunostainers (Table 2). Endogenous peroxidase activity was blocked by a 10 -min treatment with $3 \%$ hydrogen peroxide in distilled water. To minimize background staining, sections were preincubated with normal goat serum (BioGenex) for monoclonal antibodies. Slides were incubated with primary antibodies for $30 \mathrm{~min}$ to $1 \mathrm{~h}$. Immunoperoxide staining was accomplished using the super sensitive streptavidin-biotin detection kit (BioGenex) or universal avidin-biotin Ventana DAB detection system (Ventana, Phoenix, AZ). Counterstain was then performed using hematoxylin or methyl green prior to coverslipping and viewing by light microscopy.

All slides were reviewed by a single histopathologist who was blinded to clinical outcomes (AK). For ER, PR, VEGF, EGF-R, HER2/neu, and p53, slides were assessed for the percentage of tumor cells positive for each stain as well as a subjective assessment of intensity of staining, graded from 0 to $3+$. An IHC score was then calculated for each slide by multiplying the percentage of cells stained by a factor corresponding to the intensity score ( 2 for $1+$ staining, 3 for $2+$ staining, and 4 for $3+$ staining). For CD31 and CD105, microvessel counts were performed at $200 \times$ magnification within designated 'hotspots' (areas containing the greatest microvessel density), and counted over at least three high-powered fields per slide. The highest count from each specimen was reported. For Ki-67, percentage of cells stained was reported. Quantification of TSP-1 expression was performed using a computer-assisted image analysis (CAS 2000 two-color system; Becton Dickinson, San Jose, CA). Analysis was carried out by measuring the optical density of tumor specimens at $400 \times$ magnification; multiple fields were examined within each specimen and values were averaged.

\section{Statistics}

Clinical status at last follow-up was initially examined as an ordered discrete variable: alive without disease, alive with recurrence, or dead of disease. Two patients who died of other causes were excluded

Table 2. Immunohistochemical (IHC) antibodies

\begin{tabular}{|c|c|c|c|c|c|c|}
\hline Antibody & Manufacturer & Clone & IHC stainer & Dilution & Antigen retrieval & $\mathrm{AB}$ incubation time (min) \\
\hline ER & NovoCastra (Newcastle upon Tyne, UK) & 6F11 & NexES & $1: 15$ & MW & 32 \\
\hline PR & BioGenex, San Ramon, CA & PR88 & I6000 & $1: 80$ & MW & 60 \\
\hline P53 & Santa Cruz (Santa Cruz, CA) & DO-1 & NexES & $1: 200$ & MW & 32 \\
\hline HER2/neu & NeoMarkers (Fremont, CA) & E2-4001 & NexES & $1: 150$ & MW & 32 \\
\hline EGF-R & NeoMarkers (Fremont, CA) & 111.6 & NexES & $1: 400$ & Protease $8 \mathrm{~min}$ & 32 \\
\hline VEGF & Santa Cruz & C-1 & NexES & $1: 40$ & Protease $12 \mathrm{~min}$ & 32 \\
\hline Ki67 & Immunotech (Westbrook, ME) & MIB-1 & I6000 & $1: 200$ & MW & 60 \\
\hline CD31 & DAKO (Carpinteria, CA) & $\mathrm{JC} / 70 \mathrm{~A}$ & NexES & $1: 40$ & Protease $12 \mathrm{~min}$ & 32 \\
\hline CD105 & DAKO (Carpinteria, CA) & SN6h & $\mathrm{I} 6000$ & $1: 50$ & Proteinase $\mathrm{K}$ & 60 \\
\hline TSP-1 & Immunotech & BG & I6000 & $1: 20$ & NA & 60 \\
\hline
\end{tabular}

VEGF, vascular endothelial growth factor. 
from this analysis. Ordered probit models were specified and estimated by maximum likelihood using the Stata (Stata Labs, San Mateo, CA) statistical package. First, a univariate analysis was performed of the association of each IHC marker and clinical outcome. Prior to performing multivariate analyses that included the IHC markers, a multivariate ordered probit model was specified that included age, stage, grade, and tumor type. Grade and type had no statistically significant predictive power for clinical status, and were excluded from subsequent models. A multivariate ordered probit analysis was then performed including age at diagnosis, FIGO stage of disease, as well as each IHC marker in turn. These analyses were performed first for the entire study group, and then separately for only those women with stage I disease $(n=95)$.

The data were also evaluated in a hazard framework, using both a Cox proportional hazards model and a discrete choice probit hazard model, to determine if the IHC markers were predictive of length of time to the stated clinical outcomes (recurrence or death). In these models, age and stage of disease were controlled for. For the purposes of this analysis, the two women who died of other causes were included. These analyses were also performed with the Stata ${ }^{\circledR}$ statistical package.

\section{Results}

Complete follow-up data were available on 121 patients; the other 26 patients had incomplete documentation of disease status or were lost to follow-up. Four patients had inadequate tumor volume for IHC studies; thus there were 117 patients in the study population. The mean age at diagnosis was 62.8 (range: 27-92). Only eight women (6.6\%) were receiving exogenous hormones at the time of diagnosis. Median duration of follow-up was 43 months from the time of surgery (range 5-64; mean 41.2 \pm 13.4 ). At the time of last contact, 101 were alive with no evidence of disease, six were alive with known recurrence, eight were dead of disease, and two were dead of other causes. Of the 14 women who had recurrent disease or died of their malignancies, five had an initial diagnosis of FIGO stage I cancer, one had stage II, seven had stage III, and one had stage IV disease.

One hundred and five tumors had endometrioid histology; ten were serous, and two were mucinous. The tumors were graded according to WHO standards; of the endometrioid and mucinous cancers, 57 were grade 1 , three were grade 2 , and 47 were grade 3. All patients were assigned FIGO stages based on intraoperative and pathologic findings. Ninety-one patients had stage I disease, ten had stage II, 15 had stage III, and one had stage IV disease (Table 3 ).

The most frequent primary surgical procedure was radical abdominal hysterectomy with pelvic lymph node sampling $(n=51,44 \%)$. Thirty-seven women had simple hysterectomy and lymph node sampling (32\%). Thirteen women underwent vaginal hysterectomy and lymph node sampling (11\%), and three women underwent Schauta with lymph node sampling (2.5\%). An additional 13 women underwent either simple abdominal or vaginal hysterectomy without lymphadenectomy (11\%). The vast majority of women had a concomitant bilateral salpingooophorectomy (98\%).

Thirty-eight women (32\%) received no adjuvant therapy. Thirty-four women (29\%) received postoperative radiation therapy alone, $16(14 \%)$ received chemotherapy alone, and $29(25 \%)$ were treated with both radiation therapy and chemotherapy.

Statistical analysis of each of the ten IHC markers using the univariate ordered probit model revealed a significant association of increased VEGF staining with recurrence and death in univariate analyses $(P=0.017)$ (Fig. 1). For an increase in VEGF one standard deviation above the mean, the probability of recurrence increased from 4 to $8 \%$, and the probability of death of disease increased from 5 to 15\% (Table 4). However, in multivariate analyses, only known predictors of outcome (age and FIGO stage of disease) were significantly associated with poorer prognosis. These findings did not differ between the entire study group and the subgroup with stage I disease.

Additionally, we evaluated a panel of markers, combining p53, TSP1, CD31. This 'angiogenesis index' was not significantly predictive of clinical outcome in either univariate or multivariate analysis.

Survival analyses using the Cox proportional hazards model and the discrete choice probit model

Table 3. Tumor characteristics

\begin{tabular}{lc}
\hline Tumor histology & \\
Endometrioid & $105(90 \%)$ \\
Mucinous & $2(1.7 \%)$ \\
Serous & $10(8.5 \%)$ \\
Tumor grade & \\
1 & $57(53.3 \%)$ \\
2 & $3(2.8 \%)$ \\
3 & $47(43.9 \%)$ \\
FIGO stage & \\
Stage I & $91(78 \%)$ \\
Stage II & $10(8.5 \%)$ \\
Stage III & $15(12.8 \%)$ \\
Stage IV & $1(0.9 \%)$ \\
\hline
\end{tabular}




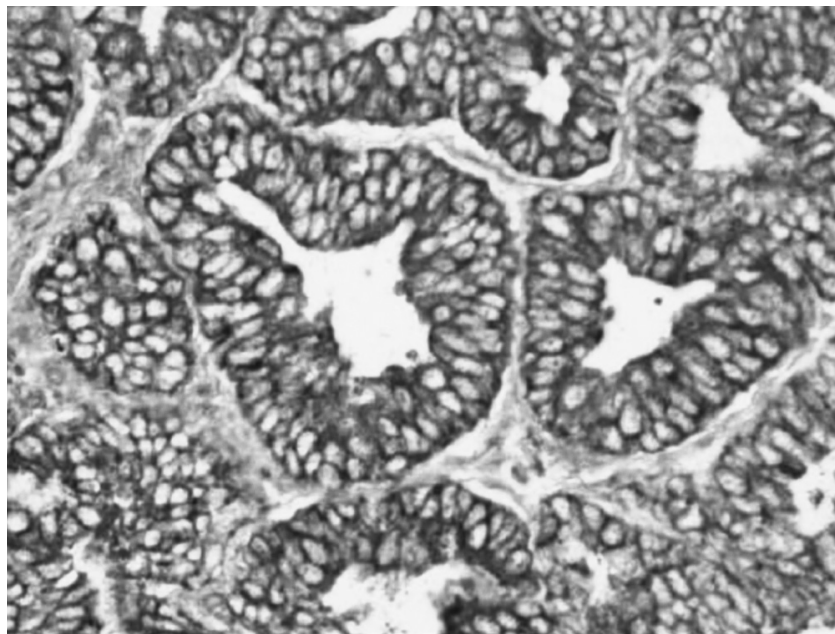

Fig. 1. Strong $(3+)$ staining of malignant endometrial glands for vascular endothelial growth factor.

revealed a statistically significant association of increased VEGF staining with likelihood of recurrence or death $(P=0.026$ and 0.011 in each model, respectively.) For an increase in VEGF staining one standard deviation above the mean, the probability of recurrence or death increased from $3.5 \%$ per year (the risk of negative clinical outcome in the study population per 12 -month period) to $6.2 \%$ per year. A decrease in VEGF staining one standard deviation below the mean decreased the probability of negative clinical outcomes to $0.8 \%$ per year. However, when multivariate survival analyses were performed including age and stage of disease, no IHC marker significantly predicted negative clinical outcome.

\section{Discussion}

Numerous prior investigators have examined IHC markers in endometrial carcinoma both as identifiers of more aggressive disease and as potential targets for adjuvant therapies ${ }^{(4,5,7-13,15,16)}$. The failure of the majority of these studies to demonstrate significant prognostic value for IHC markers may, in part, reflect the small numbers of patients included and the heterogeneity of their diseases. Most previous studies have included women at all FIGO stages of disease. Those studies which do limit their analyses to only stage I disease have included unusual histologic types such as serous and clear cell carcinomas. Endometrial cancers of these cell types are known to have more malignant behaviors. Some of the major strengths of this study are the large sample size, the relative homogeneity of the pathologic diagnoses, and the ability to separately analyze outcomes for women with stage I disease. Women with later stages of disease or more aggressive histology are known a priori to have a high risk of progression and/or recurrence. Our study addresses the clinically more meritorious question of which women with early stage endometrioid carcinoma are at highest risk to progress. This has particular clinical relevance as $90 \%$ of all women with endometrial carcinomas have endometrioid histology.

The finding that increased cellular VEGF expression in univariate analyses was predictive of poor clinical outcome is provocative. VEGF has been shown to regulate endometrial angiogenesis throughout the normal menstrual cycle, and is implicated in angiogenesis of endometrial carcinoma ${ }^{(8,9)}$. It has also been shown, in one study, to correlate with unfavorable clinical outcomes in patients with endometrial carcinoma $^{(8)}$. The lack of significance in our multivariate analyses may be a result of the relatively low number of patients with negative clinical outcomes in our patient cohort. It is possible that, with a larger number of recurrences and/or deaths, this finding would persist in multivariate analyses.

Statistical power, in fact, is the greatest weakness of the current study. While the total number of patients evaluated was large, relatively few experienced the negative outcomes of interest, decreasing the statistical power of the study to identify predictors of these outcomes. This distribution of clinical outcomes is not surprising; the majority of our study patients had stage I disease, and 5-year survival for stage I endometrial carcinoma is repeatedly reported as being approximately $85 \%{ }^{(4,25)}$.

Another possible confounder in the present study is that many of the IHC markers studied correlated with known prognostic variables, such as stage or grade, and with each other (eg, ER and PR.) This may affect the study results in two ways. Firstly, those IHC markers that correlated with other predictors of prognosis or with other markers are not truly independent

Table 4. Predictive probability of vascular endothelial growth factor staining in univariate ordered probit analysis

\begin{tabular}{llll}
\hline Immunohistochemical score & Alive & Recurrent cancer & Dead of disease \\
\hline 150 (mean -1 SD) & 0.98 & 0.01 & 0.01 \\
300 (mean) & 0.91 & 0.04 & 0.05 \\
450 (mean +1 SD) & 0.77 & 0.08 & 0.15 \\
\hline
\end{tabular}


variables, and the use of an ordered probit analysis may be misleading. Secondly, those patients with higher grade or stage tumors are more likely to have received adjuvant therapy, which might attenuate the course of the disease, or prevent the 'sought' negative clinical outcomes. Therefore, those markers that correlated with advanced stage or grade may, in fact, have a greater correlation with outcome than was seen, because patients overexpressing these markers received different therapy that positively affected the course of their disease (prevented negative outcomes).

While the present study did not clearly identify an IHC marker capable of distinguishing 'high-risk' early endometrial carcinoma, we are continuing to search for a molecular marker(s) that better serve this purpose. Meaningful and adequately powered IHC evaluation of large numbers of early stage endometrial cancers might be advanced by the use of tumor banking and by cooperative investigations among multiple institutions. Ultimately, we believe that molecular profiling of individual patients' tumors, to better prognosticate and to tailor therapy, may allow for more specific and less toxic therapies.

\section{Acknowledgments}

This work was supported by an unrestricted educational grant from Oncotech, Inc. This study was presented at the annual meeting of the Western Association of Gynecologic Oncologists, June 15, 2002, Newport Beach, CA.

\section{References}

1 American Cancer Society. Cancer Facts and Figures 2002, Atlanta GA: American Cancer Society; 2002.

2 Burris H. Docetaxel (taxotere) plus trastuzumab (Herceptin) in breast cancer. Semin Oncol 2001;28 (s3):38-44.

3 Hortobagyi GN. Overview of treatment results with trastuzumab (Herceptin) in metastatic breast cancer. Semin Oncol 2001;28 (6 S18):43-7.

4 Gehrig PA, Van Le L, Olatidoye B, Geradts J. Estrogen receptor status, determined by immunohistochemistry, as a predictor of the recurrence of stage I endometrial carcinoma. Cancer 1999;86:2083-9.

5 Sivridis E, Giatromanolaki A, Koukourakis M, Anastasiadis P. Endometrial carcinoma: association of steroid hormone receptor expression with low angiogenesis and bcl-2 expression. Virchow's Arch 2001;438:470-7.

6 Deligdisch L, Kase N, Bleiweiss I. Endometrial cancer in elderly women: a histologic and steroid receptor analysis. Gerontology 2000;46:17-21.

7 Khalifa M, Mannel R, Haraway S, Walker J, Min K. Expression of EGF-R, HER-2/neu, P53, and PCNA in endometrioid, serous papillary, and clear cell endometrial adenocarcinomas. Gynecol Oncol 1994;53:84-92.

8 Hirai M, Nakagawara A, Oosaki T, Hayashi Y, Horon M, Yoshihara T. Expression of vascular endothelial growth factors (VEGF-A/VEGF-1 and VEGF-C/VEGF-2) in postmenopausal uterine endometrial carcinoma. Gynecol Oncol 2001;80:181-8.

9 Seki N, Kodama J, Hongo A, Miyagi Y, Yoshinouchi M, Kudo T. Vascular endothelial growth factor and plateletderived endothelial growth factor expression are implicated in the angiogenesis of endometrial cancer. Eur J Cancer 2000;36:68-73.

10 Fine BA, Valente PT, Feinstein GI, Dey T. VEGF, flt-1, and $\mathrm{KDR} / \mathrm{flk}-1$ as prognostic indicator in endometrial carcinoma. Gyn Oncol 2000;76:33-9.

11 Miturski R, Semczuk A, Postawski K, Jakowicki J. Epidermal growth factor receptor immunostaining and epidermal growth factor receptor-tyrosine kinase activity in proliferative and neoplastic human endometrium. Tumor Biol 2000;21:358-66.

12 Giatromanolaki A, Sivridis E, Koukourakis M, Harris A, Gatter K. Bcl-2 and p53 expression in stage I endometrial carcinoma. Anticancer Res 1998;18:3689-94.

13 Özsaran A, Türker S, Dikmen Y et al. p53 staining as a prognostic indicator in endometrial carcinoma. Eur J Gynaec Oncol 1999;20:156-9.

14 Ramondetta L, Palazzo J, Dunton C, Kovatich A, Carlson J. A comparative analysis of $\mathrm{Ki}-67, \mathrm{p} 53$, and $\mathrm{p} 21^{\mathrm{WAF}^{1} \mathrm{CIP}^{1}}$ expression in tamoxifen associated endometrial carcinomas. Anticancer Res 1998;18:4661-6.

15 Coppola D, Fu L, Nicosia S, Kounelis S, Jones M. Prognostic significance of p53, bcl-2, vimentin and S100 protein-positive langerhans cells in endometrial carcinoma. Hum Pathol 1998;29:455-62.

16 Seki N, Kodama J, Hashimoto I, Hongo A, Yoshinouchi M, Kudo T. Thrombospondin-1 and -2 messenger RNA expression in normal and neoplastic endometrial tissues: correlation with angiogenesis and prognosis. Int J Oncol 2001;19:305-10.

17 Wang SC. Targeting HER2: recent developments and future directions for breast cancer patients. Semin Oncol 2001;28 (6 S18): 21-9.

18 Emi Y. Metastatic breast cancer with HER2/neu positive cells tends to have a morbid prognosis. Surgery 2002;131: S217-21.

19 Mirza AN. Prognostic factors in node-negative breast cancer: a review of studies with sample size more than 200 and follow up more than 5 years. Ann Surg 2002;235: 10-26.

20 Meden H, Kuhn W. Overexpression of the oncogene c-erbB-2 (HER2/neu) in ovarian cancer: a new prognostic factor. Eur J Obstetr Gynecol Reprod Biol 1997;71: 173-9.

21 Sapino A, Bongiovanni M, Cassoni P et al. Expression of CD31 by cells of extensive ductal in situ and invasive carcinomas of the breast. J Pathol 2001;194:254-61.

22 Kumar S, Ghellal A, Li C et al.Breast carcinoma: vascular density determined using CD105 antibody correlates with tumor prognosis. Cancer Res 1999;59:856-61.

$23 \mathrm{Li} \mathrm{C}$, Guo B, Bernabeu C, Kumar S. Angiogenesis in breast cancer: the role of transforming growth factor $\beta$ and CD105. Microsc Res Tech 2001;52:437-49. 
24 Albo D, Rothman V, Roberts D, Tuszynski G. Tumour cell thrombospondin-1 regulates tumour cell adhesion and invasion through the urokinase plasminogen activator receptor. Br J Cancer 2000;83:298-306.

25 DiSaia PJ, Creasman WT. Adenocarcinoma of the uterus. In: DiSaia, PJ, Creasman, WT, eds. Clinical Gynecologic
Oncology, 6th edn. St. Louis, MO: Mosby-Year Book, Inc. 2002, 137-71.

Accepted for publication October 14, 2003 